Public Lead Awareness and Responsibility.

Since current compliance with CDC recommended guidelines for blood lead screening is not universally appropriate, an increased public and parent awareness of the hazards and the symptoms and signs of lead poisoning, especially in children, should be encouraged. (Millichap JG. Environmental Poisons in Our Food. Chicago, PNB Publ, 1993).

\title{
DIAZINON EXPOSURE AND INFANTILE HYPERTONIA
}

A 12-week-old infant girl who developed persistent hypertonicity 5 weeks following exposure to the organophosphate insecticide diazinon (KnoxOut 2FM) in the home is reported from Oregon State University, Corvallis, OR. The infant's urine contained alkylphosphate metabolites of diazinon (60 ppb diethylphosphate and $20 \mathrm{ppb}$ diethylthiophosphate). Serum cholinesterase was normal. Diazinon levels in the home (floor, vacuum cleaner dust, and air) were excessive even at 6 months after application. Six weeks after evacuating the home, the infant's muscle tone returned to normal, ankle clonus had resolved, and subsequent development was normal. (Wagner SL, Orwick DL. Chronic organophosphate exposure associated with transient hypertonia in an infant. Pediatrics July 1994;94:94-97). (Reprints: Dr Sheldon L Wagner, Agricultural Chemistry, Oregon State University, Agricultural \& Life Sciences 1007, Corvalllis, OR 97331).

COMMENT. None of the typical muscarinic or nicotinic symptoms of organophosphate intoxication was present in this infant.

Organophosphates can cause a delayed neurobehavioral toxicity, characterized by neuritis, paralysis, and psychological changes, the result of degeneration of myelin and nerve axons and effects on neurotransmitters. A relationship between Parkinson's disease and exposure to pesticide chemicals has been demonstrated in agricultural workers. Children presenting with unexplained neurobehavioral symptoms should be investigated for possible exposure to insecticide environmental toxins.

\section{MOVEMENT DISORDERS}

\section{HEMIFACIAL SPASMS AND CEREBELLAR ANGLE TUMORS}

Two children, aged 3 years, with hemisomatic spasms caused by tumors in the ipsilateral cerebellopontine angle are reported from the Division of Pediatric Neurology and Department of Neurology, University of Texas Southwestern Medical Center, Dallas, TX. Patient 1 had persistent hemifacial spasms with onset soon after birth; some were complicated by flexion of the arm and extension of the leg. An initial diagnosis of partial seizures was not confirmed by video-EEG monitor, and anticonvulsants were of no benefit. CT and ultrasound were normal, but MRI revealed a C-P angle tumor. Following partial resection of a low-grade ganglioneuroma, the spasms were less severe. Patient 2 developed left sided jerks at 2 years of age, turning of the head to the right, and flexion of left elbow and hip, without loss of consciousness. Movements were worse while speaking or watching television. The EEG was normal and carbamazepine without benefit. MRI uncovered a left sided angle tumor, and spasms ceased after partial resection of a ganglioneuroma. (AlShahwan SA, Roach ES et al. Hemisomatic spasms in children. Neurology July 
1994;44:1332-1333). (Reprints: Dr ES Roach, Department of Neurology, University of Texas Southwestern Medical Center, 5323 Harry Hines Blvd, Dallas, TX 75235).

COMMENT. The absence of impaired consciousness, normal EEG, and lack of response to antiepileptic drugs should help to distinguish hemifacial or hemisomatic spasms from partial epilepsy and lead to confirmation of a posterior fossa tumor by MRI.

\section{TOURETTE'S SYNDROME AND STIMULUS-INDUCED TICS}

Three patients with Tourette's syndrome (TS) and tic-related behaviors induced by external and internal stimuli are reported from the Department of Psychiatry, University College Medical School, Middlesex Hospital, London. All young adults whose tics began in childhood had developed a variety of obsessive compulsive disorders, sensory tics, reflex motor tics, or exaggerated startle responses, in response to internal (tightness in the chest) and external (people coughing or spitting) stimuli. The term "impulsions" has been used to describe these stimulus-induced behaviors which overlap with reflex tics and sensory tics. (Eapen V, Moriarty J, Robertson MM. Stimulus induced behaviours in Tourette's syndrome. L Neurol Neurosurg Psychiatry July 1994;57:853-855).

(Respond: Dr Robertson, Department of Psychiatry, Middlesex Hospital, Mortimer Street, London W1N 8AA, England).

COMMENT. The authors use "reflex tic" to describe those in response to external stimuli (someone coughing), and "sensory tic" for those induced by sensations (tingling) felt in the soma or an internal stimulus (oneself coughing). The overlap between these various induced behaviors makes their separation difficult.

\section{CLOMIPRAMINE FOR COMPULSIVE TICS}

Reduction of adventitious movements and compulsions during clomipramine treatment (25 $\mathrm{mg}-200 \mathrm{mg}$ daily at bedtime) in five prepubertal, autistic, retarded boys is reported from the Division of Child and Adolescent Psychiatry, Bellevue Hospital and New York University Medical Center, New York. Ratings were conducted before, after 2 and 4 weeks treatment, and every 4 weeks. All three classes of movements responded to medication: general dyskinesia, akathisia, and tics. Medications were administered in a nonblind trial in clinical emergencies to extremely disturbed patients with severe environmental and familial stresses. (Brasic JR et al. Clomipramine ameliorates adventitious movements and compulsions in prepubertal boys with autistic disorder and severe mental retardation. Neurology July 1994;44:13091312). (Reprints: Dr James R Brasic, Department of Psychiatry, New York University School of Medicine, 550 First Avenue, New York, NY 10016).

COMMENT. Medicated autistic patients frequently have akathisia and tics. The differentiation of various adventitious movements in a heterogeneous group of patients is difficult, and specificity of response to treatment is limited.

\section{CHOREIFORM MOVEMENTS AND VALPROIC ACID}

Three patients, aged 10,17, and 36 years, who developed chorea during long-term treatment with valproic acid are reported from the Comprehensive Epilepsy Program, Bowman Gray School of Medicine, Winston-Salem, NC. All patients had severe brain damage, one had a vascular lesion in the caudate 